\title{
Uncertainty Theory Based Novel Multi-Objective Optimization Technique Using Embedding Theorem with Application to R \& D Project Portfolio Selection
}

\author{
Rupak Bhattacharyya, Amitava Chatterjee, Samarjit Kar \\ Department of Mathematics, National Institute of Technology, \\ Durgapur, India \\ E-mail: \{mathsrup,amitavamath\}@gmail.com,kar_s_k@yahoo.com \\ Received June 6, 2010; revised July 19, 2010; accepted July 22, 2010
}

\begin{abstract}
This paper introduces a novice solution methodology for multi-objective optimization problems having the coefficients in the form of uncertain variables. The embedding theorem, which establishes that the set of uncertain variables can be embedded into the Banach space $C[0,1] \times C[0,1]$ isometrically and isomorphically, is developed. Based on this embedding theorem, each objective with uncertain coefficients can be transformed into two objectives with crisp coefficients. The solution of the original m-objectives optimization problem with uncertain coefficients will be obtained by solving the corresponding $2 \mathrm{~m}$-objectives crisp optimization problem. The R \& D project portfolio decision deals with future events and opportunities, much of the information required to make portfolio decisions is uncertain. Here parameters like outcome, risk, and cost are considered as uncertain variables and an uncertain bi-objective optimization problem with some useful constraints is developed. The corresponding crisp tetra-objective optimization model is then developed by embedding theorem. The feasibility and effectiveness of the proposed method is verified by a real case study with the consideration that the uncertain variables are triangular in nature.
\end{abstract}

Keywords: Uncertainty Theory, Uncertain Variable, Embedding Theorem, $\alpha$-Optimistic and $\alpha$-Pessimistic Value, R \& D Project Portfolio Selection

\section{Introduction}

An important problem in topology is to decide when a space $X$ can be embedded into another space $Y$, i.e., when there exists an embedding from $X$ into $Y$. This problem is called embedding problem. Theorems asserting the embedding of a space into some other space which is more manageable than the original space are known as embedding theorems. On the other hand, a theorem which asserts that a certain space cannot be embedded into some other space is known as non-embedding theorems. Nonembedding theorems are often quite deep and require methods well beyond the general topology. For example it is by no means trivial to prove that the 2 -sphere $\left(S^{2}\right)$ cannot be embedded into the Euclidean space.

The embedding theorems in crisp and fuzzy environments are already established. The fuzzy embedding theorem shows that each fuzzy number can be identified isometrically and isomorphically with an element in $C[0$,
$1] \times C[0,1]$ where $C[0,1]$ is the set of all real valued continuous functions on $[0,1]$. Puri and Ralescu [1] and Kaleva [2] have proved that the set of all fuzzy numbers can be embedded into a Banach space isometrically and isomorphically. $\mathrm{Wu}$ and $\mathrm{Ma}$ [3] provide a specific Banach space, which shows that the set of all fuzzy numbers can be embedded into the Banach space $C[0,1] \times$ $C[0,1]$. Wu [4] propose an $(\alpha, \beta)$-optimal solution concept of fuzzy optimization problem based on the possibility and necessity measures. To do so, the fuzzy optimization problem is transformed into a bi-objective programming problem by applying the embedding theorem. $\mathrm{Wu}$ [5] shows that the optimal solution of the crisp optimization problem obtained from the fuzzy optimization problem by using embedding theorem is also an optimal solution of the original fuzzy optimization problem under the set of core values of fuzzy numbers.

With increasing competition and limitations of financial resources, the way of selection of R \& D projects 
that maximize some measure of utility or benefit to the organization has become a critical one. The purposes of project portfolio decision are to allocate a limited set of resources to projects in a way that balance risk, reward and alignment with corporate strategy. Poor selection of $\mathrm{R} \& \mathrm{D}$ projects could have a significantly negative impact on organizations for decades. The $\mathrm{R} \& \mathrm{D}$ project portfolio decision deals with future events and opportunities, much of the information required to make portfolio decisions is at best uncertain and at worst very unreliable. Project selection is usually described in term of constraint optimization problem. Given a set of project proposals, the goal is to select a subset of projects to maximize some objective without violating the constraints. An R \& D project usually involves several phases. Therefore, each phase is an option that is contingent on earlier of other options. Some attempts already exist for R \& D project portfolio selection. Rabbani et al. [6,7], Fang et al. [8], Riddell and Wallace [9], Eilat et al. [10], Stummer and Heidenberger [11], Linton et al. [12], Ringuest et al. [13], Schmidt [14] and others have done significant works in the field of R \& D project portfolio selection. To model uncertainty and vagueness, fuzzy set theory is used by many to characterize uncertain R \& D project information. Pereira and junior [15], Coffin and Taylor [16], Machacha and Bhattacharyya [17], Kuchta [18], Mohamed and McCowan [19], Hsu et al. [20], Wang and Hwang [21], Kim et al. [22], Karsak [23] have applied fuzzy set theory in the field of R \& D project portfolio selection. Unfortunately, R \& D project managers have been unable to adopt many of these mechanisms.

But in reality, sometimes investors have to deal with the uncertainty which acts neither randomness nor fuzziness. In order to deal with such type of uncertainty, Liu [24] founds uncertainty theory as a branch of mathematics. Subsequently, Liu [25] proposes uncertain process and uncertain differential equation to deal with dynamic uncertain phenomena. In addition, uncertain calculus is introduced by Liu [26] to describe the function of uncertain processes, uncertain inference is introduced by Liu [26] via the tool of conditional uncertainty and uncertain logic is proposed by Li and Liu [27] to deal with uncertain knowledge. Liu [28] proposes an uncertain programming including expected value model, chance constrained programming and dependent-chance programming to model several optimization problems. Till now, several research works $[29,30]$ have been done in this area, but none has considered the $\mathrm{R} \& \mathrm{D}$ project portfolio selection problem in the uncertain environment. Basically, till date, no embedding theorem based optimization technique is proposed in uncertainty theory.

In this paper, in Section 2, we provide some prelimi- naries required to develop the paper. In Section 3, an uncertain embedding theorem is proved and an uncertain single/multiple objective optimization method using the embedding theorem is established. In Section 4, we develop an uncertain linear bi-objective $\mathrm{R} \& \mathrm{D}$ project portfolio selection model. The objectives are 1) maximization of project benefit and 2) minimization of project risk. The risk is defined as the maximum loss that the decision maker may face in the worst case. This is considered as the projected maximum loss in case of failure of the project. Constraints on budget and resources are also considered. Using the embedding theorem established in Section 3, we convert the bi-objective uncertain optimization problem into a tetra-objective crisp optimization problem which is further transformed into a deterministic convex optimization model by global criteria approach. In Section 5 of this paper a real case study is provided to illustrate our method. The optimization software LINGO is used for the simulation. Finally in Section 6 some concluding remarks are presented.

\section{Preliminaries}

Before discussing the embedding theorem and its relevance in uncertain optimization problem we would like to discuss some basic concepts related with metric space, topology and uncertainty theory.

Definition 2.1 (Metric Space) A non-empty set $X$ is said to be a metric space if to every pair of elements $x, y$ of this set, there corresponds a non-negative real number $\rho(x, y)$ for which the following conditions hold.

1) $\rho(x, y)>0$ and $\rho(x, y)=0$ if and only if $x=y$

2) $\rho(x, y)=\rho(y, x)$

3) for any three elements $x, y, z$ in $X$,

$$
\rho(x, y) \leq \rho(x, z)+\rho(z, y) .
$$

The number $\rho(x, y)$ is called the difference or metric between the elements $\mathbf{x}, \mathbf{y}$ and the above three conditions are called metric axioms and a metric space is sometimes written as $(X, \rho)$.

Definition 2.2 A sequence $\left\{x_{n}\right\}$ of elements of a metric space $X$ is called a Cauchy sequence if for every $\varepsilon>0$ there exists a positive integer $N$ such that $\rho\left(x_{n}, x_{m}\right)<$ $\varepsilon$ whenever $n, m \geq N$.

If every Cauchy sequence of a metric space $X$ has a finite limit in $X$ then $X$ is called complete. By Cauchy's general principle of convergence it can be shown that the real line and the complex plane with usual metric are the complete metric spaces.

Definition 2.3 A set $E$ is called a normed linear space if

1) $E$ is a linear space with real or complex numbers as scalars and

2) to every element $x$ of $E$ there is associated a unique 
real number, called the norm of the element $x$ and denoted by $\|x\|$.

The norm of an element $x$ has to satisfy the following axioms.

1) $\|x\|>0$ and $\|x\|=0$ if and only if $x=\theta$

2) $\|\alpha x\|=|\alpha|\|x\|$ where $\alpha$ is a scalar

3) $\|x+y\| \leq\|x\|+\|y\|$ for every $x, y$ in $E$.

Note: In a normed linear space we can introduce a metric by $\rho(x, y)=\|x-y\|$. The metric axioms are fulfilled as

1) $\rho(x, y)>0$ and $\rho(x, y)=0$ if and only if $\|x-y\|=$ 0 ,i.e., if and only if $x-y=\theta$, i.e., if and only if $x=y$.

2) $\rho(x, y)=\|x-y\|=\|(-1)(y-x)\|=|-1|\|y-x\|=$ $\|y-x\|=\rho(y, x)$

3) $\rho(x, y)=\|x-y\|=\|(x-z)+(z-x)\| \leq\|x-z\|+$ $\|z-y\|=\rho(x, z)+\rho(z, y)$.

Definition 2.4 (Banach Space) If a normed linear space is complete in the sense of the convergence in norm, then it is called a Banach space.

Every finite dimensional normed linear space $E$ is complete (that is a Banach space) and bounded. Every finite dimensional linear space can be made a Banach space.

Definition 2.5 Two objects $A$ and $B$ are said to be congruent (or isometric) if there exists a bijection $f: A \rightarrow B$ which preserves all distances in the sense that $d(x, y)=d$ $(f(x), f(y))$ for all pairs $(x, y)$ of points in $A, d$ being used to denote the distance between points. Such a bijection, when it exists, is called congruence (or an isometry).

Definition 2.6 Let $(X, \tau),(Y, \Psi)$ be topological spaces. An embedding (or imbedding) theorem of $X$ into $Y$ is a function $e: X \rightarrow Y$ which is a homeomorphism when considered as a function from $(X, \tau)$ onto $(e(X), Y / e(X))$.

Definition 2.7 A function $e: X \rightarrow Y$ is an embedding function if and only if it is continuous and one-one and for every open set $V$ in $X$ there exists an open subset $W$ of $Y$ such that $e(V)=W \cap Y$.

Definition 2.8 The space $C[0,1]$ is the set of all real valued continuous functions $f$ on $[0,1]$, such that $f$ is left-continuous for any $t \in(0,1]$ and right-continuous at 0 , and $f$ has a right limit for any $t \in[0,1)$. The norm is defined as $\|f\|=\sup _{\mathrm{t} \in[0,1]}|f(t)|$.

Definition 2.9 Let $\Gamma$ be a non-empty set and $A$ a $\sigma$-algebra over $\Gamma$. Each element $\Lambda \in \AA$ is called an event. Let $M$ be a set function over $\AA$. Then $M$ is called an uncertain measure (Liu, [24]) if it satisfies the following four axioms.

Axiom 1. (Normality) $M\{\Gamma\}=1$;

Axiom 2. (Monotonicity) $M\left\{\Lambda_{1}\right\} \leq M\left\{\Lambda_{2}\right\}$ whenever $\Lambda_{1} \subset \Lambda_{2}$;

Axiom 3. (Self-Duality) $M\{\Lambda\}+M\left\{\Lambda^{c}\right\}=1$ for any event $\Lambda$;

Axiom 4. (Countable Subadditivity)
$M\left\{\bigcup_{i=1}^{\infty} \Lambda_{i}\right\} \leq \sum_{i=1}^{\infty} M\left\{\Lambda_{i}\right\}$, for every countable sequence of events $\left\{\Lambda_{i}\right\}$.

The triplet $(\Gamma, \AA, M)$ is called an uncertainty space.

Definition 2.10 An uncertain variable is a measurable function $\bar{\xi}$, from an uncertain space $(\Gamma, \AA, M)$ to the set of all real numbers, i.e., for any Borel set $B$ of real numbers, the set $\{\breve{\xi} \in B\}=\{\gamma \in \Gamma \mid \bar{\xi}(\gamma) \in B\}$ is an event.

Definition 2.11 (Liu, [24]) An uncertain variable $\breve{\xi}$ is said to have a first identification function $\lambda$ if

1) $\lambda(x)$ is a non-negative function on $\mathbb{R}$ such that $\sup _{x \neq y}(\lambda(x)+\lambda(y))=1$;

2) for any set $B$ of real numbers, we have,

$$
M\{\bar{\xi} \in B\}=\left\{\begin{array}{cc}
\sup _{x \in B} \lambda(x) & \text { if } \sup _{x \in B} \lambda(x)<0.5 \\
1-\sup _{x \in B^{c}} \lambda(x) & \text { if } \sup _{x \in B} \lambda(x) \geq 0.5 .
\end{array}\right.
$$

Definition 2.12 A rectangular uncertain variable is defined to be the uncertain variable which is fully determined by the pair $(a, b)$ of crisp numbers with $a<b$, and whose first identification function is

$$
\lambda(x)=0.5, \mathrm{a} \leq \mathrm{x} \leq \mathrm{b} .
$$

Definition 2.13 A triangular uncertain variable is defined to be the uncertain variable which is fully determined by the triplet $(a, b, c)$ of crisp numbers with $a<b$ $<c$, and whose first identification function is

$$
\lambda(x)= \begin{cases}\frac{x-a}{2(b-a)} & \text { if } \mathrm{a} \leq \mathrm{x} \leq \mathrm{b} \\ \frac{c-x}{2(c-b)} & \text { if } \mathrm{b} \leq \mathrm{x} \leq \mathrm{c} .\end{cases}
$$

Definition 2.14 A trapezoidal uncertain variable is defined to be the uncertain variable which is fully determined by the quadruplet $(a, b, c, d)$ of crisp numbers with $a<b<c<d$, and whose first identification function is

$$
\lambda(x)=\left\{\begin{array}{cc}
\frac{x-a}{2(b-a)} & \text { if } \mathrm{a} \leq \mathrm{x} \leq \mathrm{b} \\
0.5 & \text { if } \mathrm{b} \leq \mathrm{x} \leq \mathrm{c} \\
\frac{\mathrm{d}-x}{2(d-c)} & \text { if } \mathrm{c} \leq \mathrm{x} \leq \mathrm{d} .
\end{array}\right.
$$

Definition 2.15 (Liu, [24]) An uncertain variable $\breve{\xi}$ is said to have a second identification function $\rho$ if

1) $\rho(x)$ is a nonnegative and integrable function on $\mathbb{R}$ such that $\int_{\mathbb{R}} \rho(x) d x \geq 1$;

2) for any set $B$ of real numbers, we have,

$$
M\{\xi \in B\}=\left\{\begin{array}{cc}
\int_{B} \rho(x) d x & \text { if } \int_{B} \rho(x) d x<0.5 \\
1-\int_{B^{c}} \rho(x) d x & \text { if } \int_{B} \rho(x) d x \geq 0.5 .
\end{array}\right.
$$


Definition 2.16 An exponential uncertain variable is defined to be the uncertain variable having the second identification function

$$
\rho(x)=\frac{1}{\beta} \exp \left(-\frac{x}{\alpha}\right), x \geq 0,
$$

and is denoted by $\operatorname{EXP}(\alpha, \beta)$, where $\alpha, \beta$ are real numbers with $\alpha \geq \beta>0$. Note that $\int_{-\infty}^{\infty} \rho(x) d x=\frac{\alpha}{\beta} \geq 1$.

Definition 2.17 A bell-shaped uncertain variable is defined to be the uncertain variable having the second identification function

$$
\rho(x)=\frac{1}{\beta \sqrt{\pi}} \exp \left(-\frac{(x-m)^{2}}{\alpha^{2}}\right), x \in \mathbb{R},
$$

and is denoted by $B(m, \alpha, \beta)$, where $\alpha, \beta$ are real numbers with $\alpha \geq \beta>0$. Note that $\int_{0}^{\infty} \rho(x) d x=\frac{\alpha}{\beta} \geq 1$.

Definition 2.18 (Liu, [24]) The uncertain variables $\breve{\xi}_{1}, \breve{\xi}_{2}, \ldots, \breve{\xi}_{n}$ are said to be independent if

$$
M\left\{\bigcap_{i=1}^{n}\left\{\breve{\xi}_{i} \in B_{i}\right\}\right\}=\min _{1 \leq i \leq n} M\left\{\breve{\xi}_{i} \in B_{i}\right\}
$$

for Borel sets $B_{1}, B_{2}, \ldots, B_{n}$ of real numbers.

Definition 2.19 (Liu, [24]) The uncertainty distribution $\Phi: \mathbb{R} \rightarrow[0,1]$ of an uncertain variable $\breve{\xi}$ is defined by

$$
\Phi(\mathrm{x})=\mathrm{M}\{\breve{\xi} \leq \mathrm{x}\} .
$$

Definition 2.20 An uncertain variable $\bar{\xi}$ is called normal if its distribution function $\Phi$ is given by

$$
\Phi(x)=\left(1+\exp \left(\frac{\pi(m-x)}{\sqrt{3} \sigma}\right)\right)^{-1}, x \in \mathbb{R} .
$$

It is then denoted by $N(m, \sigma)$, where $m, \sigma(>0)$ are real numbers.

Definition 2.21 (Chen [8]) Let $\bar{\xi}$ be an uncertain variable and $\alpha \in(0,1]$. Then

$$
\breve{\xi}_{o p t}^{\alpha}=\sup \{r \mid M\{\breve{\xi} \geq r\} \geq \alpha\}
$$

is called the $\alpha$-optimistic value of $\xi$, and

$$
\breve{\xi}_{p e s}^{\alpha}=\inf \{r \mid M\{\breve{\xi} \geq r\} \geq \alpha\}
$$

is called the $\alpha$-pessimistic value of $\bar{\xi}$.

Example 2.22 Let $\breve{\xi}=(a, b)$ be a rectangular uncertain variable. Then its $\alpha$-optimistic and $\alpha$-pessimistic values are

$$
\breve{\xi}_{\text {opt }}^{\alpha}=\left\{\begin{array}{ll}
b & \text { if } \alpha \leq 0.5 \\
a & \text { if } \alpha>0.5,
\end{array} \quad \xi_{\text {pes }}^{\alpha}= \begin{cases}b & \text { if } \alpha \leq 0.5 \\
a & \text { if } \alpha>0.5\end{cases}\right.
$$

Example 2.23 Let $\bar{\xi}=(a, b, c)$ be a triangular uncertain variable. Then its $\alpha$-optimistic and $\alpha$-pessimistic values are

$$
\begin{aligned}
& \xi_{\text {opt }}^{\alpha}=\left\{\begin{array}{cc}
2 \alpha b+(1-2 \alpha) c & \text { if } \alpha \leq 0.5 \\
(2 \alpha-1) a+(2-2 \alpha) b & \text { if } \alpha>0.5,
\end{array}\right. \\
& \breve{\xi}_{\text {pes }}^{\alpha}=\left\{\begin{array}{cc}
(1-2 \alpha) a+2 \alpha b & \text { if } \alpha \leq 0.5 \\
(2-2 \alpha) b+(2 \alpha-1) c & \text { if } \alpha>0.5 .
\end{array}\right.
\end{aligned}
$$

Example 2.24 Let $\bar{\xi}=(a, b, c, d)$ be a trapezoidal uncertain variable. Then its $\alpha$-optimistic and $\alpha$-pessimistic values are

$$
\begin{aligned}
& \breve{\xi}_{\text {opt }}^{\alpha}=\left\{\begin{array}{cc}
2 \alpha c+(1-2 \alpha) d & \text { if } \alpha \leq 0.5 \\
(2 \alpha-1) a+(2-2 \alpha) b & \text { if } \alpha>0.5,
\end{array}\right. \\
& \breve{\xi}_{\text {pes }}^{\alpha}=\left\{\begin{array}{cc}
(1-2 \alpha) a+2 \alpha b & \text { if } \alpha \leq 0.5 \\
(2-2 \alpha) c+(2 \alpha-1) d & \text { if } \alpha>0.5 .
\end{array}\right.
\end{aligned}
$$

Example 2.25 Let $\bar{\xi}=\operatorname{EXP}(a, b)$ be an exponential uncertain variable. Then its $\alpha$-optimistic and $\alpha$-pessimistic values are

$$
\begin{aligned}
& \breve{\xi}_{\text {opt }}^{\alpha}= \begin{cases}a \cdot \ln \left(\frac{a}{a-\alpha b}\right) & \text { if } \alpha<0.5 \\
a \cdot \ln \left(\frac{a}{b-\alpha b}\right) & \text { if } \alpha \geq 0.5,\end{cases} \\
& \breve{\xi}_{p e s}^{\alpha}=\left\{\begin{array}{cc}
a \cdot \ln \left(\frac{a}{\alpha b}\right) & \text { if } \alpha<0.5 \\
a \cdot \ln \left(\frac{a}{a-(1-\alpha) b}\right) & \text { if } \alpha \geq 0.5 .
\end{array}\right.
\end{aligned}
$$

Example 2.26 Let $\bar{\xi}=\mathrm{B}(e, a, b)$ be a bell-shaped uncertain variable. Then its $\alpha$-optimistic and $\alpha$-pessimistic values are

$$
\begin{aligned}
& \breve{\xi}_{\text {opt }}^{\alpha}=\left\{\begin{array}{cr}
\frac{a}{\sqrt{2}} \Phi^{-1}\left(\frac{\alpha b}{a}\right)+e & \text { if } \alpha<0.5 \\
\frac{a}{\sqrt{2}} \Phi^{-1}\left(\frac{a-(1-\alpha) b}{a}\right)+e & \text { if } \alpha \geq 0.5,
\end{array}\right. \\
& \breve{\xi}_{p e s}^{\alpha}= \begin{cases}\frac{a}{\sqrt{2}} \Phi^{-1}\left(\frac{a-\alpha b}{a}\right)+e & \text { if } \alpha<0.5 \\
\frac{a}{\sqrt{2}} \Phi^{-1}\left(\frac{(1-\alpha) b}{a}\right)+e & \text { if } \alpha \geq 0.5 .\end{cases}
\end{aligned}
$$

Example 2.27 Let $\breve{\xi}=\aleph(m, \sigma)$ be a normal uncertain variable. Then its $\alpha$-optimistic and $\alpha$-pessimistic values are

$$
\breve{\xi}_{\text {opt }}^{\alpha}=m-\frac{\sqrt{3} \sigma}{\pi} \ln \left(\frac{\alpha}{1-\alpha}\right), \breve{\xi}_{p e s}^{\alpha}=m+\frac{\sqrt{3} \sigma}{\pi} \ln \left(\frac{\alpha}{1-\alpha}\right) .
$$

Theorem 2.28 Let $\bar{\xi}$ be an uncertain variable. Then $\xi_{o p t}^{\alpha}$ is an increasing and left continuous function of $\alpha$. 
Also $\breve{\xi}_{p e s}^{\alpha}$ is a decreasing and left-continuous function of $\alpha$ (Liu [24]).

\section{Uncertain Multiple Objective Optimization Method Using Embedding Theorem}

In this section, we introduce a solution methodology for multiple objective programming problems in uncertain environment by using the concept of optimistic and pessimistic values of uncertain variables.

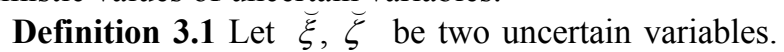
We write $\breve{\xi} \succeq \breve{\zeta}$ if and only if

$$
\breve{\xi}_{\text {opt }}^{\alpha} \geq \breve{\zeta}_{\text {opt }}^{\alpha}, \breve{\xi}_{\text {pes }}^{\alpha} \geq \breve{\zeta}_{\text {pes }}^{\alpha} \forall \alpha \in[0,1] \text {. }
$$

We also write $\breve{\xi} \preceq \breve{\zeta}$ if and only if $\breve{\zeta} \succeq \breve{\xi}$.

On the other hand, we write that $\breve{\xi} \succ \breve{\zeta}$ if and only if

$$
\begin{gathered}
\breve{\xi}_{o p t}^{\alpha} \geq \breve{\zeta}_{o p t}^{\alpha}, \breve{\xi}_{p e s}^{\alpha}>\breve{\zeta}_{p e s}^{\alpha} \forall \alpha \in[0,1] \text { or, } \\
\breve{\xi}_{\text {opt }}^{\alpha}>\breve{\zeta}_{\text {opt }}^{\alpha}, \breve{\xi}_{p e s}^{\alpha} \geq \breve{\zeta}_{p e s}^{\alpha} \forall \alpha \in[0,1] \text { or, } \\
\breve{\xi}_{\text {opt }}^{\alpha}>\breve{\zeta}_{\text {opt }}^{\alpha}, \breve{\xi}_{p e s}^{\alpha}>\breve{\zeta}_{p e s}^{\alpha} \forall \alpha \in[0,1] .
\end{gathered}
$$

We also write $\breve{\xi} \preceq \zeta$ if and only if $\breve{\zeta} \prec \breve{\xi}$.

Definition 3.2 Let $\bar{\xi}$ be an uncertain variable. Then the norm of $\bar{\xi}$ is defined by $\|\breve{\xi}\|=E|\breve{\xi}|$.

Justification:

$$
\begin{aligned}
& \text { 1) }\|\breve{\xi}+\breve{\eta}\|=E|\breve{\xi}+\breve{\eta}| \\
& =\int_{0}^{\infty} M\{|\breve{\xi}+\breve{\eta}| \geq r\} d r \\
& \leq\left[\int_{0}^{\infty} M\{|\breve{\xi}| \geq r / 2\} d r+\int_{0}^{\infty} M\{|\breve{\eta}| \geq r / 2\} d r\right] \\
& =[2 E|\breve{\xi}|+2 E|\breve{\eta}|]=2\|\breve{\xi}\|+2\|\breve{\eta}\|
\end{aligned}
$$

(Triangular inequality as defined by Liu [24]).

$$
\text { 2) } \| c \xi|=E| c \xi)=|c| E|\xi|=|c|\|\xi\| \text {. }
$$

Theorem 3.3 (Embedding theorem) Let the function $\pi: U(\mathbb{R}) \rightarrow C[0,1] \times C[0,1]$ is defined by

$$
\pi(\breve{\xi})=\left\{\begin{array}{l}
\left(\breve{\xi}_{\text {opt }}^{\alpha}, \breve{\xi}_{p e s}^{\alpha}\right), \text { if } \alpha>0.5 \\
\left(\breve{\xi}_{p e s}^{\alpha}, \breve{\xi}_{\text {opt }}^{\alpha}\right), \text { if } \alpha \leq 0.5
\end{array}\right.
$$

Then the following properties hold good.

1) $\pi$ is injective.

2) $\pi((1\{\mathrm{~s}\} \times \breve{\xi})+(1\{\mathrm{t}\} \times \breve{\eta}))=\mathrm{s} \pi(\breve{\xi})+\mathrm{t} \pi(\breve{\eta}) \forall \bar{\xi}, \breve{\eta} \in$ $U(\mathbb{R}), s \geq 0, t \geq 0$.

3) $d_{U}(\breve{\xi}, \breve{\eta})=\|\pi(\breve{\xi})-\pi(\breve{\eta})\|$.

That is, $U(\mathbb{R})$ can be embedded into $C[0,1] \times C[0,1]$ isometrically and isomorphically.

Proof. Let $\alpha \leq 0.5$.

1) Let, if possible, $\bar{\xi}, \breve{\zeta}$ be two distinct uncertain

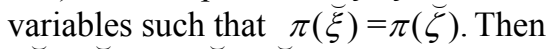
$\left(\breve{\xi}_{\text {pes }}^{\alpha}, \breve{\xi}_{\text {opt }}^{\alpha}\right)=\left(\breve{\zeta}_{\text {pes }}^{\alpha}, \breve{\zeta}_{\text {opt }}^{\alpha}\right)$.

Since the two real open intervals are equal, the corresponding boundary points must be the same. Then $\xi_{p e s}^{\alpha}=\breve{\zeta}_{p e s}^{\alpha}$ and $\breve{\xi}_{\text {opt }}^{\alpha}=\breve{\zeta}_{\text {opt }}^{\alpha}$, which contradicts the fact that $\bar{\xi} \neq \zeta$. Hence our assumption is wrong and consequently the mapping $\pi$ is injective.

2) We have the bottom equation.

3) We have,

$$
\begin{aligned}
& d_{U}(\xi, \eta)=E|\xi-\eta| \\
& =E\left(|\xi-\eta|_{\text {pes }}^{\alpha},|\xi-\eta|_{\text {opt }}^{\alpha}\right)=\left(E\left(|\xi-\eta|_{\text {pes }}^{\alpha}\right), E\left(|\xi-\eta|_{\text {opt }}^{\alpha}\right)\right) \\
& =\left(\|\xi-\eta\|_{\text {pes }}^{\alpha},\|\xi-\eta\|_{\text {opt }}^{\alpha}\right)=(\pi\|\xi-\eta\|)=\|\pi(\xi)-\pi(\eta)\| .
\end{aligned}
$$

For $\alpha>0.5$ the proof is similar.

Proposition 3.4 (Order preserving) Let $\pi$ be the function defined in theorem 3.3 and let $\xi, \zeta \in U(\mathbb{R})$. Then $\xi \preceq \zeta$ if and only if $\pi(\xi) \leq \pi(\zeta)$. We also have $\xi \prec \zeta$ if and only if $\pi(\xi)<\pi(\zeta)$.

Proof. We note that

$$
\begin{gathered}
\xi \leq \zeta \Leftrightarrow \xi_{\text {opt }}^{\alpha} \leq \zeta_{\text {opt }}^{\alpha}, \xi_{\text {pes }}^{\alpha}<\zeta_{p e s}^{\alpha} \forall \alpha \in[0,1] \\
\Leftrightarrow\left(\xi_{\text {opt }}^{\alpha}, \xi_{p e s}^{\alpha}\right) \leq\left(\zeta_{\text {opt }}^{\alpha}, \zeta_{p e s}^{\alpha}\right) \forall \alpha \in\left[0, \frac{1}{2}\right], \\
\left(\xi_{p e s}^{\alpha}, \xi_{\text {opt }}^{\alpha}\right) \leq\left(\zeta_{\text {pes }}^{\alpha}, \zeta_{\text {opt }}^{\alpha}\right) \forall \alpha \in\left[\frac{1}{2}, 1\right] \\
\Leftrightarrow \pi(\xi) \leq \pi(\zeta) .
\end{gathered}
$$

Similarly it can be shown that $\xi<\zeta$ if and only if $\pi(\xi)<\pi(\zeta)$.

Definition 3.5 Let $\breve{f}_{1}, \breve{f}_{2}, \breve{g}_{1}, \breve{g}_{2}$ be real valued functions defined by $\breve{f}_{1}, \breve{f}_{2}, \breve{g}_{1}, \breve{g}_{2}: V \rightarrow U(\mathbb{R})$, where $V$ is a real vector space. We say that $\left(\breve{f}_{1}, \breve{g}_{1}\right) \preceq\left(\breve{f}_{2}, \breve{g}_{2}\right)$ if and

$$
\begin{aligned}
& \pi((1\{s\} \times \xi)+(1\{t\} \times \zeta)) \\
& =\left([(1\{s\} \times \xi)+(1\{t\} \times \zeta)]_{p e s}^{\alpha},\left[((1\{s\} \times \xi)+(1\{t\} \times \zeta)]_{o p t}^{\alpha}\right)\right. \\
& =\left(\left[(1\{s\} \times \xi)_{p e s}^{\alpha}+(1\{t\} \times \zeta)_{p e s}^{\alpha}\right],\left[(1\{s\} \times \xi)_{o p t}^{\alpha}+(1\{t\} \times \zeta)_{o p t}^{\alpha}\right]\right) \\
& =\left(\left([1\{s\}]_{p e s}^{\alpha} \cdot \xi_{p e s}^{\alpha}+[1\{t\}]_{p e s}^{\alpha} \cdot \zeta_{p e s}^{\alpha}\right),\left([1\{s\}]_{o p t}^{\alpha} \cdot \xi_{o p t}^{\alpha}+[1\{t\}]_{o p t}^{\alpha} \cdot \zeta_{o p t}^{\alpha}\right)\right. \\
& =\left(s \cdot \xi_{p e s}^{\alpha}+t \cdot \zeta_{p e s}^{\alpha}, s \cdot \xi_{o p t}^{\alpha}+t \cdot \zeta_{o p t}^{\alpha}\right)=s \pi(\xi)+t \pi(\zeta) .
\end{aligned}
$$


only if $\breve{f}_{1} \preceq \breve{f}_{2}, \breve{g}_{1} \preceq \breve{g}_{2}$. We say that $\left(\breve{f}_{1}, \breve{g}_{1}\right) \prec\left(\breve{f}_{2}, \breve{g}_{2}\right)$ if and only if $\forall \alpha \in[0,1]$,

$\left(\breve{f}_{1}\right)_{o p t}^{\alpha} \leq\left(\breve{g}_{1}\right)_{o p t}^{\alpha},\left(\breve{f}_{2}\right)_{\text {pes }}^{\alpha}<\left(\breve{g}_{2}\right)_{\text {pes }}^{\alpha}$

or, $\left(\breve{f}_{1}\right)_{o p t}^{\alpha}<\left(\breve{g}_{1}\right)_{\text {opt }}^{\alpha},\left(\breve{f}_{2}\right)_{\text {pes }}^{\alpha} \leq\left(\breve{g}_{2}\right)_{\text {pes }}^{\alpha}$

or, $\left(\breve{f}_{1}\right)_{\text {opt }}^{\alpha}<\left(\breve{g}_{1}\right)_{\text {opt }}^{\alpha},\left(\breve{f}_{2}\right)_{\text {pes }}^{\alpha}<\left(\breve{g}_{2}\right)_{\text {pes }}^{\alpha}$.

Let $\breve{f}$ be a function defined by $\breve{f}: V \rightarrow U(\mathbb{R})$. Let $g_{i}(x), i=1,2, \ldots, m$ be real-valued functions defined on the same real vector space $V$ and $X$ be any subspace of $V$. Then let us now consider the following optimization problem as follows.

$$
\left\{\begin{array}{cc}
\min & \breve{f}(x) \\
\text { subject to } & g_{i}(x) \leq 0, \quad i=1,2, \ldots, m, \\
& x \in X .
\end{array}\right.
$$

Then $x^{*}$ is an optimal solution of the problem (3.1) if there exists no $x\left(\neq x^{*}\right)$ such that $\breve{f}(x) \prec \breve{f}\left(x^{*}\right)$.

Let $\pi$ be the function defined in theorem 3.3. Now we consider the following optimization problem by applying the embedding function $\pi$ to problem (3.1).

$$
\left\{\begin{array}{cc}
\min & \pi(\breve{f}(x)) \\
\text { subject to } & g_{i}(x) \leq 0, \quad i=1,2, \ldots, m . \\
& x \in X .
\end{array}\right.
$$

Then $x^{*}$ is an optimal solution of the problem (3.2) if there exists no $x\left(\neq x^{*}\right)$ such that $\pi(\breve{f}(x))<\pi\left(\breve{f}\left(x^{*}\right)\right)$.

Therefore, $x^{*}$ is an optimal solution of the problem (3.2) if there exists no $x\left(\neq x^{*}\right)$ such that

$\left(\left(\breve{f}(x)_{\text {pes }}^{\alpha},\left(\breve{f}(x)_{\text {opt }}^{\alpha}\right)<\left(\left(\widetilde{f}\left(x^{*}\right)_{\text {pes }}^{\alpha},\left(\breve{f}\left(x^{*}\right)_{\text {opt }}^{\alpha}\right)\right.\right.\right.\right.$, (follow definition (3.1).

Proposition 3.6 $x^{*}$ is an optimal solution of the problem (3.1) if and only if $x^{*}$ is an optimal solution of the problem (3.2).

Proof. Proposition 3.4 states that $\breve{f}(x) \prec \breve{f}\left(x^{*}\right)$ if and only if $\pi(\breve{f}(x))<\pi\left(\breve{f}\left(x^{*}\right)\right)$, and so, the proof is obvious.

Therefore, the optimal solution of problem (3.1) is same as the optimal solution of the following problem:

$$
\left\{\begin{array}{cc}
\min & \left\{\breve{f}(x)_{p e s}^{\alpha}, \breve{f}(x)_{o p t}^{\alpha}\right\} \\
\text { subject to } & g_{i}(x) \leq 0, \\
& x \in X .
\end{array} \quad i=1,2, \ldots, m .\right.
$$

Proposition 3.7 If $x^{*}$ is a Pareto optimal solution of the problem (3.3) for some $\alpha^{*} \in[0,1]$, then $x^{*}$ is an optimal solution of the problem (3.2).

Proof. Since $x^{*}$ is a Pareto optimal solution of the problem (3.3), $x^{*}$ is a feasible solution of the problem (3.2). If possible, let $x^{*}$ is not an optimal solution of problem (3.2). Then there exists a feasible solution $x(\neq$ $\left.x^{*}\right)$ such that $\pi(\breve{f}(x))<\pi\left(\breve{f}\left(x^{*}\right)\right) \forall \alpha \in[0,1]$.

Then $\forall \alpha \in[0,1]$, we have

either $\breve{f}(x)_{p e s}^{\alpha} \leq \breve{f}\left(x^{*}\right)_{p e s}^{\alpha}, \breve{f}(x)_{o p t}^{\alpha}<\breve{f}\left(x^{*}\right)_{o p t}^{\alpha}$

or, $\breve{f}(x)_{p e s}^{\alpha}<\breve{f}\left(x^{*}\right)_{p e s}^{\alpha}, \breve{f}(x)_{o p t}^{\alpha} \leq \breve{f}\left(x^{*}\right)_{o p t}^{\alpha}$

or, $\breve{f}(x)_{p e s}^{\alpha}<\breve{f}\left(x^{*}\right)_{p e s}^{\alpha}, \breve{f}(x)_{o p t}^{\alpha}<\breve{f}\left(x^{*}\right)_{o p t}^{\alpha}$.

Since $\alpha^{*} \in[0,1]$, we then should have, either

$\breve{f}(x)_{p e s}^{\alpha^{*}} \leq \breve{f}\left(x^{*}\right)_{p e s}^{\alpha^{*}}, \breve{f}(x)_{o p t}^{\alpha^{*}}<\breve{f}\left(x^{*}\right)_{o p t}^{\alpha^{*}} \mathrm{~s}$

or, $\breve{f}(x)_{p e s}^{\alpha^{*}}<\breve{f}\left(x^{*}\right)_{p e s}^{\alpha^{*}}, \breve{f}(x)_{o p t}^{\alpha^{*}} \leq \breve{f}\left(x^{*}\right)_{o p t}^{\alpha^{*}}$

or, $\breve{f}(x)_{p e s}^{\alpha^{*}}<\breve{f}\left(x^{*}\right)_{p e s}^{\alpha^{*}}, \breve{f}(x)_{o p t}^{\alpha^{*}}<\breve{f}\left(x^{*}\right)_{o p t}^{\alpha^{*}}$.

This shows that $x^{*}$ is not an optimal solution of the problem (3.3); a contradiction with the assumption that it is a pareto-optimal solution of (3.3). So, our assumption is wrong and we are with the theorem.

Theorem 3.8 If $x^{*}$ is a Pareto optimal solution of the problem (3.3) for some $\alpha^{*} \in[0,1]$, then $x^{*}$ is an optimal solution of the problem (3.1).

Proof. The theorem is obvious from proposition 3.6 and 3.7.

Now we consider the uncertain multi-objective optimization problem as follows:

$$
\left\{\begin{array}{l}
\min \left(\breve{f}_{1}(x), \breve{f}_{2}(x), \ldots ., \breve{f}_{n}(x)\right) \\
\operatorname{subject~to~} g_{j}(x) \leq 0, j=1,2, \ldots, m \\
x \in X
\end{array}\right.
$$

$$
\left\{\begin{aligned}
& \min \left\{\pi\left(\breve{f}_{1}(x), \breve{f}_{2}(x), \ldots . ., \breve{f}_{n}(x)\right)\right\} \\
&= \min \left\{\pi\left(\breve{f}_{1}(x)\right), \pi\left(\breve{f}_{2}(x)\right), \ldots \ldots, \pi\left(\breve{f}_{n}(x)\right)\right\} \\
&= \min \left[\left\{\breve{f}_{1}(x)_{\text {pes }}^{\alpha}, \breve{f}_{1}(x)_{\text {opt }}^{\alpha}\right\},\left\{\breve{f}_{2}(x)_{\text {pes }}^{\alpha}, \breve{f}_{2}(x)_{\text {opt }}^{\alpha}\right\}, \ldots \ldots . .,\right. \\
&\left.\quad\left\{\breve{f}_{n}(x)_{\text {pes }}^{\alpha}, \breve{f}_{n}(x)_{\text {opt }}^{\alpha}\right\}\right] \\
&= \min \left[\breve{f}_{1}(x)_{p e s}^{\alpha}, \breve{f}_{1}(x)_{o p t}^{\alpha}, \breve{f}_{2}(x)_{p e s}^{\alpha}, \breve{f}_{2}(x)_{o p t}^{\alpha}, \ldots \ldots ., \breve{f}_{n}(x)_{p e s}^{\alpha}, \breve{f}_{n}(x)_{o p t}^{\alpha}\right] \\
& \operatorname{subject~to~} g_{j}(x) \leq 0, j=1,2, \ldots, m \\
& x \in X, 0 \leq \alpha \leq 1
\end{aligned}\right.
$$


We say that $x^{*}$ is an optimal solution of problem (3.4) if there exists no $x \neq x^{*}$ such that $\tilde{f}_{i}(x) \prec\left(\tilde{f}_{i}\left(x^{*}\right)\right.$.

Let $\pi$ be the embedding function defined in theorem 3.3. Then we consider the multi-objective optimization problem (3.5) by applying the embedding function $\pi$ to problem (3.4).

We say that $x^{*}$ is an optimal solution of problem (3.5), if there exists no $x \neq x^{*}$ such that $\pi\left(\breve{f}_{i}(x)\right)<\pi\left(\left(\breve{f}_{i}\left(x^{*}\right)\right)\right.$.

Theorem 3.9 If $x^{*}$ is a Pareto optimal solution of (3.5) for some $\alpha^{*} \in[0,1]$, then $x^{*}$ is an optimal solution of the uncertain multi-objective optimization problem (3.4).

Note: To solve uncertain multi-objective problem by using embedding theorem we first have to transform the uncertain multi-objective optimization problem into the crisp multi-objective optimization problem (3.5). The Pareto optimal solution of this problem is the optimal solution of the original uncertain multi-objective problem.

\section{Uncertain R \& D Project Portfolio Selection model}

In this section, we first describe the notations used in the construction of the $\mathrm{R} \& \mathrm{D}$ project portfolio selection model. Then the objective function of the models will be constructed in the second subsection. In the third subsection we will discuss the constraints used in our portfolio selection model. The next subsection will include final mathematical model.

\subsection{Notations}

$N=$ Number of candidate projects.

$T=$ Number of periods.

$I=$ Interest rate.

$x_{i t}=\left\{\begin{array}{l}1 \text { if project } \mathrm{i} \text { is selected in period } t \\ 0 \text { otherwise }\end{array}\right.$,

$x=\left(x_{i t}\right)_{N \times T}=$ decision matrix.

$\breve{v}_{i t}=$ Projected uncertain outcome of project $i$ in period $t$.

$\breve{r}_{i t}=$ Projected uncertain risk of implementing project $i$ in period $t$.

$\bar{c}_{i t}=$ Expected uncertain cost required by $i^{\text {th }}$ project in period $t$.

$B_{t}=$ Budget available for stage $t$.

$R_{i t}^{s}=$ Amount of resource of type $s$ required for implementation of project $i$ individually in period $t$.

$R_{s t}^{\prime}=$ Amount of available resources of type $s$ in period $t$.

$\bar{R}_{s}=$ Total amount of available resources of type $s$.

\subsection{Formulation of Objective Functions}

In this $\mathrm{R} \& \mathrm{D}$ project portfolio selection problem we have considered two objectives: maximization of the benefit and minimization of the project risk.

\subsubsection{Maximization of Benefit}

The total outcome from the projects will be obtained by considering the total individual. If the interest rate for each period is $I$, the total outcome is

$$
\breve{Z}_{O}(x)=\sum_{t=1}^{T} \frac{1}{(1+I)^{t}} \sum_{i=1}^{N} \breve{v}_{i t} x_{i t} .
$$

The total cost will be obtained by the total individual costs for each project. Then the total cost is

$$
\breve{Z}_{C}(x)=\sum_{i=1}^{T} \sum_{i=1}^{N} \breve{c}_{i t} x_{i t} .
$$

Thus the benefit of the project portfolio is

$$
\breve{Z}_{B}(x)=\breve{Z}_{O}(x)-\breve{Z}_{C}(x)=\sum_{i=1}^{T} \sum_{i=1}^{N}\left[\frac{1}{(1+I)^{t}} \breve{v}_{i t} x_{i t}-\breve{c}_{i t} x_{i t}\right] .
$$

Our objective will be to maximize the benefit.

\subsubsection{Minimization of Project Risk}

For successfully implementation of R\&D project portfolio, the risk attached with the projects must be as less as possible. Here, we have defined risk as the opposite of expected profit. As the futures of all the projects are uncertain, implementation of a project may or may not yield us success. In case of failure, the decision maker may loose their money and time and resource. Let $r_{i t} \geq 0$ is the amount the decision maker may loose in worst case for the $i^{\text {th }}$ project at period $t$. Then the total risk involved in the project portfolio is $\sum_{t=1}^{T} \sum_{i=1}^{N} \breve{r}_{i t} x_{i t}$.

Therefore, the objective is to minimize total risk

$$
\breve{Z}_{R}(x)=\sum_{t=1}^{T} \sum_{i=1}^{N} \breve{r}_{i t} x_{i t} .
$$

Thus we are with the following bi-objective optimization problem

$$
\begin{cases}\operatorname{Max} & \breve{Z}_{B}(x) \\ \operatorname{Min} & \breve{Z}_{\mathrm{R}}(x) .\end{cases}
$$

\subsection{Formulation of the Constraints}

In this subsection we will formulate the constraints required to model the problem realistically. 


\subsubsection{Outcome Constraints}

As the minimum expected outcome for the projects in period $t$ is $V_{t}$, we have,

$$
\sum_{i=1}^{N} \breve{v}_{i t} x_{i t} \succeq V_{t} \forall t
$$

i.e., $\sum_{i=1}^{N}\left(\breve{v}_{i t}\right)_{\text {pes }}^{\alpha} x_{i t} \geq V_{t}, \sum_{i=1}^{N}\left(\breve{v}_{i t}\right)_{\text {opt }}^{\alpha} x_{i t} \geq V_{t} \forall t$.

\subsubsection{Resource Constraints}

The projects are implemented by using limited amount of resources. As the available resources are always finite, the required resource with particular type should be within the resource available of that type for each period. Thus we have

$$
\sum_{i=1}^{N} R_{i t}^{s} x_{i t} \leq R_{s t}^{\prime} \forall s, t .
$$

The total amount of resources available is limited. So, the amount of resource required should not be more than the total resource available for each type of resources. Thus we have,

$$
\sum_{t=1}^{T}\left(\sum_{i=1}^{N} R_{i t}^{s} x_{i t}\right) \leq \bar{R}_{s} \forall s .
$$

\subsubsection{Budget Constraints}

The project expenses during the planning horizon should not exceed the predetermined budget for each stage or period. So, we have

$$
\begin{aligned}
& \sum_{i=1}^{N} \breve{c}_{i t} x_{i t} \preceq B_{t} \forall t, \\
& \text { i.e., } \sum_{i=1}^{N}\left(\breve{c}_{i t}\right)_{\text {pes }}^{\alpha} x_{i t} \leq B_{t}, \sum_{i=1}^{N}\left(\breve{c}_{i t}\right)_{\text {opt }}^{\alpha} x_{i t} \leq B_{t} \forall t .
\end{aligned}
$$

\subsection{The Set of Feasible Solutions}

In this subsection we construct the set $X$ of feasible solutions $x=\left(x_{i t}\right)_{N \times T}$. Then we have problem (4.1).

\subsection{The R \& D Project Portfolio Selection Mode $l$}

Keeping in mind the objectives and constrained obtained in the last two subsections, the R \& D project portfolio selection problem is modeled as

$$
\left\{\begin{array}{cc}
\operatorname{Max} & \breve{Z}_{B}(x) \\
\operatorname{Min} & \breve{Z}_{R}(x) \\
\text { s.t. } & x \in X .
\end{array}\right.
$$

By applying embedding theorem, the uncertain multiob jective optimization problem (4.2) is converted into the crisp multi-objective problem

$$
\left\{\begin{array}{cc}
\operatorname{Max} & \left\{\breve{Z}_{B}(x)_{\text {pes }}^{\alpha}, \breve{Z}_{B}(x)_{o p t}^{\alpha}\right\} \\
\operatorname{Min} & \left\{\breve{Z}_{R}(x)_{\text {pes }}^{\alpha}, \breve{Z}_{R}(x)_{o p t}^{\alpha}\right\} \\
\text { s.t. } & x \in X, 0 \leq \alpha \leq 1 .
\end{array}\right.
$$

The global criteria methods developed in the context of multi-objective optimization problem are really handy for obtaining the Pareto optimal solution. Let,

$$
\begin{aligned}
& B_{\text {pes }}^{+}=\max \left\{\breve{Z}_{B}(x)_{\text {pes }}^{\alpha}\right\}, B_{\text {opt }}^{+}=\max \left\{\breve{Z}_{B}(x)_{\text {opt }}^{\alpha}\right\}, \\
& R_{\text {pes }}^{+}=\min \left\{\breve{R}_{B}(x)_{\text {pes }}^{\alpha}\right\}, R_{\text {opt }}^{+}=\min \left\{\breve{R}_{B}(x)_{\text {opt }}^{\alpha}\right\}, \\
& B_{\text {pes }}^{-}=\min \left\{\breve{Z}_{B}(x)_{\text {pes }}^{\alpha}\right\}, B_{\text {opt }}^{-}=\min \left\{\breve{Z}_{B}(x)_{\text {opt }}^{\alpha}\right\}, \\
& R_{\text {pes }}^{-}=\max \left\{\breve{R}_{B}(x)_{\text {pes }}^{\alpha}\right\}, R_{\text {opt }}^{-}=\max \left\{\breve{R}_{B}(x)_{\text {opt }}^{\alpha}\right\} .
\end{aligned}
$$

Then the problem (4.3) is further converted into the following single objective convex programming problem.

$$
\left\{\begin{array}{c}
\operatorname{Min}\left[\left(\frac{B_{p e s}^{+}-\breve{Z}_{B}(x)_{p e s}^{\alpha}}{B_{p e s}^{+}-B_{p e s}^{-}}\right)^{2}+\left(\frac{B_{o p t}^{+}-\breve{Z}_{B}(x)_{o p t}^{\alpha}}{B_{o p t}^{+}-B_{o p t}^{-}}\right)^{2}+\right. \\
\left.\left(\frac{\breve{R}_{B}(x)_{p e s}^{\alpha}-R_{p e s}^{+}}{R_{p e s}^{-}-R_{p e s}^{+}}\right)^{2}+\left(\frac{\breve{R}_{B}(x)_{o p t}^{\alpha}-R_{o p t}^{+}}{R_{o p t}^{-}-R_{o p t}^{+}}\right)^{2}\right]^{\frac{1}{2}} \\
\text { such that } \quad x \in X, 0 \leq \alpha \leq 1 .
\end{array}\right.
$$

\section{Case Study}

In this section a model is developed and solved based on data from the large scale organization B. M. Enterprise, Berhampore, West Bengal, India. The R \& D wing of this organization is involved in different structural works in civil, mechanical and electrical fields. During the year 2009 the organization gets 10 project proposals from private as well as public sectors. All the proposals accompany data on the estimated outcome, estimated cost, funds, workers, budget and risk. After first round of

$$
\begin{aligned}
& X=\left\{x=\left(x_{i t}\right)_{N \times T}: \sum_{i=1}^{N}\left(\breve{v}_{i t}\right)_{p e s}^{\alpha} x_{i t} \leq V_{t}, \sum_{i=1}^{N}\left(\breve{v}_{i t}\right)_{o p t}^{\alpha} x_{i t} \leq V_{t}, \sum_{i=1}^{N} R_{i t}^{s} x_{i t} \leq R_{s t}^{\prime},\right. \\
& \left.\sum_{i=1}^{N}\left(\breve{c}_{i t}\right)_{p e s}^{\alpha} x_{i t} \leq B_{t}, \sum_{i=1}^{N}\left(\breve{c}_{i t}\right)_{o p t}^{\alpha} x_{i t} \leq B_{t}, \sum_{t=1}^{T}\left(\sum_{i=1}^{N} R_{i t}^{s} x_{i t}\right) \leq \bar{R}_{s} \forall s, t\right\}
\end{aligned}
$$


scrutiny 5 project proposals are short listed. All the five projects are scheduled over two periods and each period lasts one year. They are renamed as projects I, II, III, IV and $\mathrm{V}$ due to privacy. The estimated outcome, risk and projected cost are considered in the form of triangular uncertain variables. Interest rate is $5 \%$. The estimated data for outcome, costs, risks, funds and workers are given in Table 1.

Constraints on fund, workers and budget for each period are given in Table 2.

As discussed in Section 4, the uncertain optimization model (4.2) is constructed which is converted into the model (4.3) by using the embedding theorem. The model (4.4) is then constructed as

$$
\left\{\begin{array}{r}
\text { Min }\left[\left(\frac{16.000-\breve{Z}_{B}(x)_{p e s}^{\alpha}}{5.375}\right)^{2}+\left(\frac{18.475-\breve{Z}_{B}(x)_{o p t}^{\alpha}}{7.375}\right)^{2}\right. \\
\left.+\left(\frac{\breve{R}_{B}(x)_{p e s}^{\alpha}-2.075}{1.325}\right)^{2}+\left(\frac{\breve{R}_{B}(x)_{o p t}^{\alpha}-2.2}{1.4}\right)^{2}\right]^{\frac{1}{2}}
\end{array}\right.
$$

such that $\quad x \in X, 0 \leq \alpha \leq 1$.

The solution of the model (5.1) is done by using the
LINGO software and the obtained solution is as follows: $x_{11}=0, x_{21}=1, x_{31}=0, x_{41}=1, x_{51}=1 ; x_{12}=0, x_{22}=1$, $x_{32}=0, x_{42}=0, x_{52}=1$.

It means that B. M. Enterprise should select the $2^{\text {nd }}, 4^{\text {th }}$ and $5^{\text {th }}$ projects in $1^{\text {st }}$ stage and $2^{\text {nd }}$ and $5^{\text {th }}$ projects in the second stage to get the optimum result.

For this portfolio, the benefit is estimated as $(6.5,11.4$, 13.7) million rupees and the corresponding risk is $(1.95$, $2.4,3.2)$ million rupees.

\section{Conclusions}

This paper introduces the concept of multiple objective uncertain optimization problems. In particular, this paper concentrates on the problems where the coefficients of the decision variables are uncertain variables. To do so, we propose and prove the uncertain embedding theorem from the space of uncertain variables to the Banach space $C[0,1] \times C[0,1]$. By applying embedding theorem, each uncertain objective function can be converted into two deterministic objectives functions. The Pareto optimal solution of both the deterministic objectives is the optimal solution of the uncertain objective.

\begin{tabular}{|c|c|c|c|c|c|c|}
\hline \multicolumn{2}{|c|}{ Projects } & \multirow{2}{*}{$\frac{I}{(4,7,9)}$} & \multirow{2}{*}{$\frac{\mathrm{II}}{(1,3,5)}$} & \multirow{2}{*}{$\frac{\text { III }}{(0.2,1.4,2.8)}$} & \multirow{2}{*}{$\frac{\mathrm{IV}}{(0,1,1.4)}$} & \multirow{2}{*}{$\frac{\mathrm{V}}{(5,6,7)}$} \\
\hline Outcome & $1^{\text {st }}$ period & & & & & \\
\hline (in Million Rupees) & $2^{\text {nd }}$ period & $(7,10,12)$ & $(2,3,4)$ & $(2.5,4,5.2)$ & $(1,2,3)$ & $(6,7.5,9)$ \\
\hline \multirow{2}{*}{$\begin{array}{c}\text { Cost } \\
\text { (in Million Rupees) }\end{array}$} & $1^{\text {st }}$ period & $(1,2.2,3)$ & $(0.4,1.2,2)$ & $(0.8,1,1.2)$ & $(0.1,0.3,0.8)$ & $(2,2.5,2.9)$ \\
\hline & $2^{\text {nd }}$ period & $(2,2.8,3.7)$ & $(1.0,1.1,2)$ & $(1.5,1.7,2)$ & $(1.6,1.8,2.1)$ & $(3,4,5)$ \\
\hline \multirow{2}{*}{$\begin{array}{c}\text { Risk } \\
\text { (in Million Rupees) }\end{array}$} & $1^{\text {st }}$ period & $(0.6,0.8,1)$ & $(0.1,0.2,0.4)$ & $(0.5,0.7,0.9)$ & $(0.4,0.5,0.6)$ & $(0.35,0.4,0.5)$ \\
\hline & $2^{\text {nd }}$ period & $(0,0.4,0.5)$ & $(0.6,0.7,1)$ & $(0.4,0.5,0.6)$ & $(0.4,0.5,0.6)$ & $(0.5,0.6,0.7)$ \\
\hline \multirow{2}{*}{$\begin{array}{c}\text { Fund } \\
\text { (in Million Rupees) }\end{array}$} & $1^{\text {st }}$ period & 0.6 & 0.4 & 0.25 & 0.11 & 0.21 \\
\hline & $2^{\text {nd }}$ period & 0.9 & 0.2 & 0.21 & 0.09 & 0.2 \\
\hline \multirow{2}{*}{$\begin{array}{c}\text { Workers } \\
\text { (in numbers) }\end{array}$} & $1^{\text {st }}$ period & 31 & 15 & 20 & 21 & 16 \\
\hline & $2^{\text {nd }}$ period & 10 & 12 & 17 & 18 & 9 \\
\hline
\end{tabular}

Table 1. Estimated project data.

Table 2. Constraints.

\begin{tabular}{cccc}
\hline Category & $1^{\text {st }}$ period & $2^{\text {nd }}$ period & Total \\
\hline Outcome (in Million Rupees) & $>10.0$ & $>10.0$ & - \\
Budget (in Million Rupees) & $<8.0$ & $<9.0$ & $<0.8$ \\
Fund (in Million Rupees) & $<0.9$ & $<80$ & $<1.5$ \\
Workers (in numbers) & $<85$ & $<150$ \\
\hline
\end{tabular}


This paper also introduces a new model of $\mathrm{R} \& \mathrm{D}$ project portfolio selection by identifying project information like estimated future outcome, risk or estimated cost of the projects as uncertain variables. An uncertain bi-objective optimization model, that maximizes the benefit and minimizes the risk, is constructed. Constraints on budget, resources and outcomes are also included in the model to make it more realistic. The uncertain optimization method by embedding theorem is used to solve it. A real case study is provided for illustration.

In future, we will use the uncertain optimization approach to other real optimization problems like portfolio selection problem, supply chain management problem, poverty management problem etc.

For large data sets, meta-heuristic algorithms such as tabu search, simulated annealing, ant-colony optimization, and particle swarm optimization may be employed to solve the non-linear programming problem (4.4).

\section{References}

[1] M. L. Puri and D. A. Ralescu, "Differentials for Fuzzy Functions," Journal of Mathematical Analysis and its Application, Vol. 91, No. 2, 1983, pp. 552-558.

[2] O. Kaleva, "The Cauchy Problem for Fuzzy Differential Equations," Fuzzy Sets and Systems, Vol. 35, No. 3, 1990, pp. 389-396.

[3] C. X. Wu and M. Ma, "Embedding Problem of Fuzzy Number Space: Part I," Fuzzy Sets and Systems, Vol. 44, No. 1, 1991, pp. 33-38.

[4] C. X. Wu, "An $(\alpha, \beta)$-Optimal Solution Concept in Fuzzy Optimization Problems," Optimization, Vol. 53, No. 2, 2004, pp. 203-221.

[5] C. X. Wu, "Evaluate Fuzzy Optimization Problems Based on Bi Objective Programming Problems," Computer and Mathematics with Applications, Vol. 47, No. 5, 2004, pp. 893-902.

[6] M. Rabbani, M. A. Bajestani and G. B. Khoshkhou, "A Multi-Objective Particle Swarm Optimization for Project Selection Problem," Expert Systems with Applications, Vol. 37, No. 1, 2010, pp. 315-321.

[7] M. Rabbani, R. T. Moghaddam, F. Jolai and H. R. Ghorbani, "A Comprehensive Model for R and D Project Portfolio Selection with Zero - One Linear Goal Programming," IJE Transactions A: Basic, Vol. 19, No. 1, 2006, pp. 55-66.

[8] Y. Fang, L. Chen and M. Fukushima, "A Mixed R \& D Projects and Securities Portfolio Selection Model," European Journal of Operational Research, Vol. 185, No. 2, 2008, pp. 700-715.

[9] S. Riddell and W. A. Wallace, "The Use of Fuzzy Logic and Expert Judgment in the R \& D Project Portfolio Selection Process," Proceedings: PICMET, Portland, 2007, pp. 1228- 1238 .

[10] H. Eilat, B. Golany and A. Shtub, "Constructing and
Evaluating Balanced Portfolios of R \& D Projects with Interactions: A DEA Based Methodology," European Journal of Operational Research, Vol. 172, No. 3, 2006, pp. 1018-1039.

[11] C. Stummer and K. Heidenberger, "Interactive R\&D Portfolio Selection Considers Multiple Objectives, Project Interdependencies, and Time: A Three Phase Approach," Proceedings: PICMET, Portland, 2001, pp. 423428.

[12] J. D. Linton, S. T. Walsh, B. A. Kirchhoff, J. Morabito and M. Merges, "Selection of R \& D Projects in a Portfolio," Proceedings of IEEE Engineering Management Society, Washington, 2000, pp. 506-511.

[13] J. L. Ringuest, S. B. Graves and R. H. Case, "Conditional Stochastic Dominance in R \& D Portfolio Selection," IEEE Transactions on Engineering Management, Vol. 47, No. 4, 2000, pp. 478-484.

[14] R. L. Schmidt, "A model for R \& D Project Selection with Combined Benefit, Outcome, and Resource Interactions," IEEE Transactions on Engineering Management, Vol. 40, No. 4, 1993, pp. 403-410.

[15] O. Pereira and D. Junior, "The R \& D Project Selection Problem with Fuzzy Coefficients," Fuzzy Sets and Systems, Vol. 26, No. 3, 1988, pp. 299-316.

[16] M. A. Coffin and B. W. Taylor, "Multiple Criteria R \& D Selection and Scheduling Using Fuzzy Logic," Computers Operations Researches, Vol. 23, No. 3, 1996, pp. 207-220.

[17] L. L. Machacha and P. Bhattacharya, "A Fuzzy-LogicBased Approach to Project Selection," IEEE Transactions on Engineering Management, Vol. 47, No. 1, 2000, pp. 65-73.

[18] D. Kuchta, "A Fuzzy Mode for R \& D Project Selection with Benefit: Outcome and Resource," The Engineering Economist, Vol. 46, No. 3, 2001, pp. 164-180.

[19] S. Mohamed and A. K. McCowan, "Modelling Project Investment Decisions under Uncertainty Using Possibility Theory," International Journal of Project Management, Vol. 19, No. 4, 2001, pp. 231-241.

[20] Y. G. Hsu, G. H. Tzeng and J. Z. Shyu, "Fuzzy Multiple Criteria Selection of Government-Sponsored Frontier Technology R \& D Projects," $R$ \& D Management, Vol. 33, No. 5, 2003, pp. 539-551.

[21] J. Wang and W. L. Hwang, "A Fuzzy Set Approach for R\&D Portfolio Selection Using a Real Options Valuation Model," Omega, Vol. 35, No. 3, 2007, pp. 247-257.

[22] S. S. Kim, Y. Choi, N. M. Thang, E. R. Ramos and W. J. Hwang, "Development of a Project Selection Method on Information System Using ANP and Fuzzy Logic," World Academy of Science, Engineering and Technology, Vol. 53, No. 6, 2009, pp. 411-415.

[23] E. E. Karsak, "A Generalized Fuzzy Optimization Framework for R\&D Project Selection Using Real Options Valuation," Proceedings of ICCCSA, Berlin, Heidelberg, New York, 2006, pp. 918-927.

[24] B. Liu, "Uncertainty Theory," 2nd Edition, Springer- 
Verlag, Berlin, 2007.

[25] B. Liu, "Fuzzy Process, Hybrid Process and Uncertain Process," Journal of Uncertain Systems, Vol. 2, No. 1, 2008, pp. 3-16.

[26] B. Liu, "Some Research Problems in Uncertainty Theory," Journal of Uncertain Systems, Vol. 3, No. 1, 2009, pp. 3-10.

[27] X. Li and B. Liu, "Hybrid Logic and Uncertain Logic," Journal of Uncertain Systems, Vol. 3, No. 2, 2009, pp. 83-94.
[28] B. Liu, "Uncertainty Theory," 3rd Edition. http://orsc.edu. $\mathrm{cn} /$ liu/ut.pdf

[29] X. Gao, "Some Properties of Continuous Uncertain Measure," International Journal of Uncertainty, Fuzziness and Knowledge-Based Systems, Vol. 18, No. 4, 2007, pp. 383-390.

[30] C. You, "Some Convergence Theorems of Uncertain Sequences," Mathematical and Computer Modelling, Vol. 49, No. 3-4, 2009, pp. 482-487. 